\title{
Hepatocyte Growth Factor (HGF) Expression in High-Fat Diet Fed Rat Corpus Cavernosum. Preliminary results.
}

\author{
I. Tomada*, N. Tomada**, F. Marques***, P. Vendeira**, D. Neves**** \\ * Master student of Faculty of Nutrition and Food Sciences of Universidade do Porto, Portugal \\ ** Department of Urology of S. João Central Hospital, Porto, Portugal \\ ***Clinical Analyses Service, Faculty of Pharmacy of Universidade do Porto, Portugal \\ **** Laboratory of Molecular Cell Biology of Faculty of Medicine and IBMC of Universidade \\ do Porto, Portugal \\ inestomada@gmail.com
}

The main cause of erectile dysfunction (ED) is organic in nature, with vasculogenic etiology being predominant. Several epidemiological studies report the relationship between ED and several well-recognized cardiovascular risk factors, including atherosclerosis, diabetes, dyslipidemia, hypertension, as well as lifestyle factors, such as obesity and sedentarism [1]. Recent findings also indicate that high-fat (HF) regular intake induces endothelial dysfunction and increases ED prevalence [2]. Due to their interconnection, ED is considered equivalent to endothelial dysfunction, and it is nowadays seen as a predictive factor of atherosclerosis and cardiovascular disease (CVD) [3-4]. It is well established that the expression of some vascular growth factors is frequently diminished in corpus cavernosum (CC) of ED patients, and that its levels are particularly modified in metabolic syndrome (MetS). This syndrome combines more than three of the illnesses that prompt to vasculogenic ED: elevated blood pressure, high triglycerides, low high-density lipoprotein (HDL) cholesterol, elevated waist circumference, and insulin resistance [5-6]. Hepatocyte Growth Factor (HGF) is a pleiotropic factor with potent mitogenic and angiogenic properties, previously employed in the treatment of ischaemic members [7-8]. Interestingly, it was demonstrated that its serum levels were particularly increased in obesity and in MetS [9-11]. HGF is expressed by several organs, but as far as we know, it has never been detected in CC. In this way, we present an immunohistochemical (IH) characterization of HGF expression in HF-diet fed rat CC.

Male Wistar rats ( 2 months-old, $\mathrm{n}=30$ ) were randomly divided in two experimental groups: HFdiet treated rats until complete 4 or 6 months, and age-matched control group. HF diet contained $45 \%$ energy from fat (metabolizable energy: 4,65kcal $/ \mathrm{g}$, TestDiet ${ }^{\circledR} 58 \mathrm{~V} 8$, Purina Mills Inc., USA) in contrast to 4\% energy from fat of standard diet (metabolizable energy: 2,90kcal/g, A04 Panlab S.L., Barcelona, Spain). Body weight and food ingestion were evaluated weekly, and glycaemia and blood pressure were monitorized. Serum insulin and testosterone were quantified by RIA (Testo-RIA-CT, Biosource Europe S.A., Belgium, and Sensitive Rat Insulin RIA Kit SRI-13K, Millipore Co., USA, respectively) and lipid profile was determined by enzymatic colorimetric tests, using commercially available kits (Cholesterol, Triglycerides, HDL Cholesterol Direct, ABX Diagnostics, Bedfordshire,UK), in a autoanalyser (Cobas Mira Plus, ABX Diagnostic, Bedfordshire, UK). Rats were sacrificed by decapitation at 4 or 6 months, and penile fragments were removed, fixed in $10 \%$ buffered formaldehyde for $24 \mathrm{~h}$ and embedded in paraffin, oriented along its transversal axis. Penile sections (5um thick) were cut with a Leica RM2145 microtome (Leica Microsystems GmbH, Wetzlar, Germany) and placed on to $0,1 \%$ poly-L-lysine coated microscopy slides for IH 
analysis. Sections were deparaffinized, hydrated, treated with 3\% hydrogen peroxide in methanol to block endogenous peroxidase activity, exposed to $\mathrm{HCl} 1 \mathrm{M}$ for $30 \mathrm{~min}$ for epitope retrieval and neutralized with Borax 0,1M for $5 \mathrm{~min}$. HGF expression was detected by goat polyclonal anti-HGF $\beta$ (dilution 1/100) (Santa Cruz Biothecnology Inc, USA) followed by biotynilated secondary antibody (goat monoclonal antibody, Sigma-Aldrich Co, UK) (dilution 1/500) and streptavidin-peroxidase complex (Vectastain-Vector Laboratories Inc, Burlingame, USA) (dilution 1/200). Sections were reacted with diaminobenzidine/peroxidase (DAB/H2O2), and counterstained with hematoxylin. Sections of all experimental groups were stained with haematoxylin-eosin (HE) for morphological study. Statistical analysis was performed with Statistical Package for the Social Sciences (SPSS ${ }^{\circledR}$, version 14.0 for Windows, SPSS Inc., Chicago, Illinois), and results are expressed as means \pm standard error of mean. Probability values less than $5 \%$ were considered significant.

Although no significative differences in anthropometric and metabolic parameters studied were observed, HF-diet treated rats showed hypertriglyceridemia and lower serum levels of HDL than control animals (Table 1). HE staining (Figure 1) evidenced cavernosal vessels delimited by well-preserved endothelium supported by smooth muscle fibers, and plentiful connective tissue between cavernosal vessels in all tissue samples. Nevertheless, HF-diet fed group CC presents a structural disorganization comparatively to age-matched control group. Close to vascular spaces, several lipid-rich cells were found in 6 months rats HF-diet fed. HGF expression was observed in smooth muscle layer and also in vascular endothelium, however no marked differences were found between studied groups (Figure 2).

HF fed rat experimental model presents a great importance in studies of MetS-related DE, since endothelial dysfunction and atherosclerosis are the main etiologies of both MetS and ED. Rats treated with HF food for 4 months present a high risk of endothelial dysfunction, due to development of hypertriglyceridemia associated to HDL decrease (Table 1). This modification of serum lipids profile is a recognized marker of insulin resistance, which increases the risk of CVD and ED. The morphological study of all experimental groups corroborates biochemical results. Particularly, the presence of adipocytes around cavernosal vessels in HF-fed animals suggests atherosclerosis development which gradually leads to cavernous vascular deterioration and endothelial dysfunction, and therefore, ED. Atherosclerosis, considered as chronic vascular inflammation, induces ischemia downstream of the atheroma plaques and increases local and systemic angiogenic factors expression [12]. Recent evidences indicate that HGF may act in atherosclerosis progression and it has also been shown its expression in atheroma plaques, particularly when associated with MetS [13-15]. On the other hand, HGF cardioprotective properties are well recognized and it was even used in the treatment of ischemic members [11].

In this report, we verified HGF expression in cavernous tissue (Figure 2), however we did not find significant differences in its expression levels. In brief, we presume that the adoption of a healthy lifestyle, associated to lipid and energetic restriction could reduce the risk of endothelial dysfunction and ED. Further molecular studies are needed in order to clarify HGF role in ED progression. 


\section{References:}

[1] C. Derby et al, Urology. 56 (2000) 302.

[2] K. Esposito, D. Giugliano, Int. J. Impot. Res. 17 (2005) 391.

[3] I. Goldstein, Int. J. Impot. Res. 15 (2003) 229.

[4] A. Guay, Endocrinol. Metab. Clin. N. Am. 36 (2007) 453.

[5] K. Esposito et al, Nutr. Metab. Cardiovasc. Dis. 17 (2007) 274.

[6] M. Carnethon et al, Diabetes Care. 25 (2002) 1358.

[7] F. Bossulino et al, J. Cell. Biol. 119 (1992) 629.

[8] E. vanBelle et al, Circulation. 97 (1998) 38.

[9] J. Rehman et al, J. Am. Coll. Cardiol. 41 (2006) 1408.

[10] J. Silha et al, Int. J. Impot. Res. 29 (2006) 1308.

[11] A. Hiratsuka et al, J. Clin. Endocrinol. Metab. 90 (2006) 2927.

[12] J. Nigro et al, Endocrine Rev. 27 (2006) 242.

[13] X. Liu et al, J. Urol. 166 (2001) 354.

[14] Y. Yamamoto et al, J. Hypertens. 19 (2001) 1975.

[15] H. Ma et al, Atherosclerosis. 164 (2002) 79.

Acknowledgements:

Authors thank Dr. Conceição Gonçalves from Laboratório Nobre of Faculty of Medicine of Universidade do Porto for testosterone RIA assays.

This study was supported in part by grants of Faculty of Nutrition and Food Sciences of Universidade do Porto.

Table 1 - Metabolic parameters of rats HF diet treated and age-matched control group. Values are means \pm SEM ( $\mathrm{n}=6$ rats/group, except 6 mo HF group $\mathrm{n}=12) * \mathrm{P}<0,05$

\begin{tabular}{|c|c|c|c|c|}
\hline & \multicolumn{2}{|l|}{ Control } & \multicolumn{2}{|l|}{ HF-diet } \\
\hline & $4 \mathrm{mo}$ & $6 \mathrm{mo}$ & $4 \mathrm{mo}$ & $6 \mathrm{mo}$ \\
\hline Body Weight $(\mathrm{g})$ & $522.3 \pm 13.2$ & $632.7 \pm 20.8$ & $537.2 \pm 21.9$ & $625.3 \pm 19.9$ \\
\hline Energy Intake (Kcal/week) & $531.1 \pm 7.3 *$ & $550.3 \pm 10.8 *$ & $727.9 \pm 20.7 *$ & $644.9 \pm 10.0 *$ \\
\hline Glycaemia $(\mathrm{mg} / \mathrm{dL})$ & $135.3 \pm 4.2$ & $144.8 \pm 5.7$ & $136.8 \pm 6.6$ & $138.8 \pm 2.3$ \\
\hline Insulin $(\mathrm{ng} / \mathrm{mL})$ & $1,38 \pm 0,02 *$ & $1,47 \pm 0,04$ & $1,49 \pm 0,02 *$ & $1,35 \pm 0,03$ \\
\hline Systolic Pressure $(\mathrm{mmHg})$ & $125.3 \pm 1.8$ & $131.5 \pm 0.5$ & $103.0 \pm 3.6$ & $134.7 \pm 0.7$ \\
\hline Diastolic Pressure $(\mathrm{mmHg})$ & $75.8 \pm 2.3$ & $80.5 \pm 0.5$ & $84.0 \pm 1.9$ & $73.0 \pm 3.5$ \\
\hline Testosterone (ng/mL) & $1,84 \pm 0,25$ & $1,00 \pm 0,17$ & $4,85 \pm 1,44$ & $1,93 \pm 0,25$ \\
\hline Total cholesterol (mg/dL) & $101,8 \pm 8,1$ & $101,1 \pm 9,6$ & $91,2 \pm 2,7$ & $112,6 \pm 6,2$ \\
\hline $\mathrm{HDL}(\mathrm{mg} / \mathrm{dL})$ & $31,4 \pm 2,7$ & $33,8 \pm 2,1 *$ & $28,3 \pm 1,0$ & $29,9 \pm 0,8 *$ \\
\hline Triglycerides (mg/dL) & $257,0 \pm 24,5 *$ & $282,8 \pm 49,3$ & $165,7 \pm 18,0^{*}$ & $183,1 \pm 16,7$ \\
\hline
\end{tabular}


Fig. 1 - HE staining evidenced well preserved endothelium in cavernosal vessels delimited by smooth muscle fibers, and plentiful connective tissue between vessels in all groups. Adipocytes (black arrow) were visualized in 6 months-old HF diet fed rats. Scale bar $=50$ um.

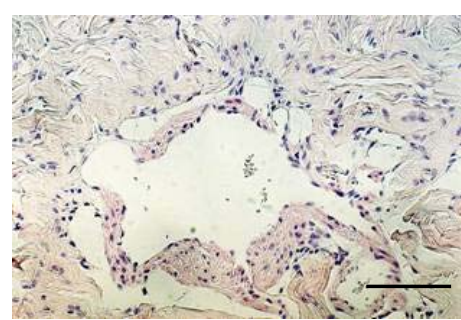

Control 4 months

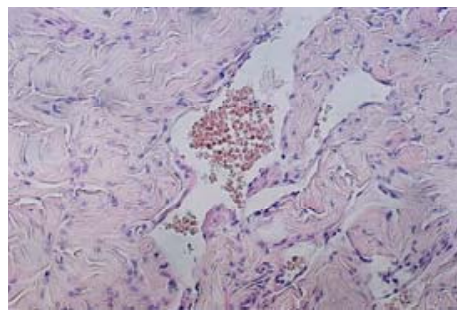

Control 6 months

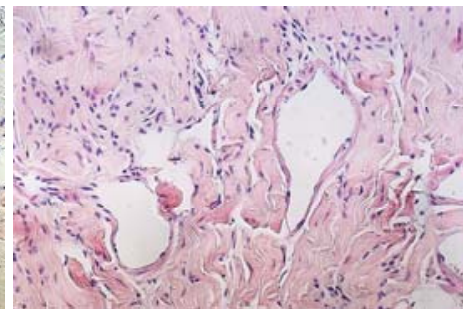

HF 4 months

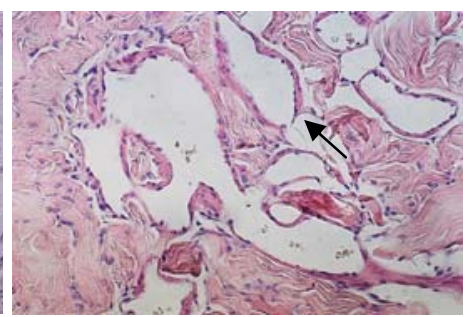

HF 6 months

Fig. 2 - HGF imunohistochemical detection reveals its expression in smooth muscle layer and also in vascular endothelium in all studied groups. No differences were observed in this growth factor expression in all experimental groups. Close to vascular spaces, several adipocytes (black arrow) were found in 6 months-old HF diet fed rats. Scale bar $=50 \mathrm{um}$.

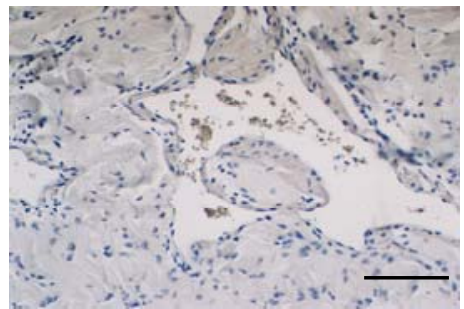

Control 4 months

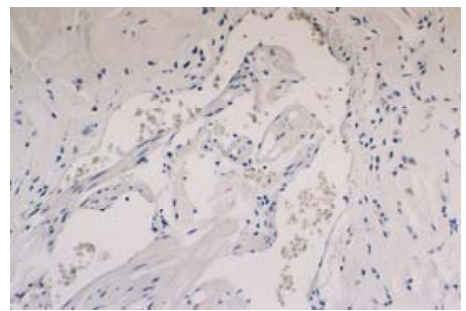

Control 6 months

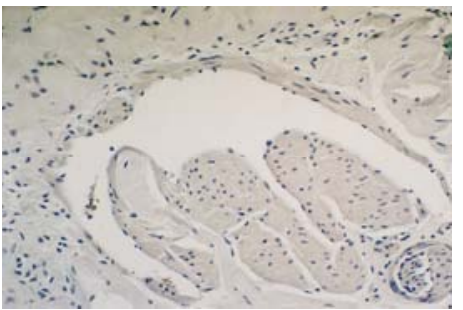

HF 4 months

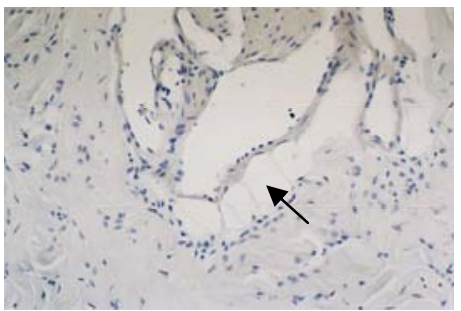

HF 6 months 